\title{
Observation of single flux quantum vortices in the intermediate state of a type-I superconducting film
}

\author{
Junyi Ge, Joffre Gutierrez, Jo Cuppens, and Victor V. Moshchalkov* \\ INPAC-Institute for Nanoscale Physics and Chemistry, KU Leuven, Celestijnenlaan 200D, B-3001 Leuven, Belgium
}

(Received 31 January 2013; revised manuscript received 24 October 2013; published 7 November 2013)

\begin{abstract}
The flux quantization in the intermediate state of a type-I superconducting $\mathrm{Pb}$ film is studied by using scanning Hall probe microscopy. The vorticity of flux tubes can be tuned by changing the cooling field through the flux expulsion process, and single flux quantum vortices coexisting with multiple quantized flux tubes are observed at low enough fields. However, the minimum fluxoid observed through flux penetration is found to contain more than one flux quantum, and its vorticity increases with decreasing temperature. By combining these two processes it is possible to stabilize flux tubes of opposite polarity, and single flux quantum vortices are created through the annihilation process under the drive of the Lorentz force. Our results give strong evidence that single quantum vortices can be thermodynamically stabilized in the intermediate state of type-I superconductors.
\end{abstract}

DOI: 10.1103/PhysRevB.88.174503

PACS number(s): 74.78.-w, 74.25.fc, 74.25.Ha, 74.25.Wx

In conventional superconductors, the formation of Cooper pairs leads to the quantization of magnetic flux in units of $\Phi_{0}=h / 2 e .{ }^{1}$ The interaction between the quantized fluxoids (vortex-vortex interaction) depends on the superconducting parameter $\kappa=\lambda / \xi$ ( $\lambda$ being the penetration depth and $\xi$ the coherence length). Until now three different kinds of vortex-vortex interactions have been observed; each of them results in the formation of different vortex patterns: (1) In the well known single-band type-II superconductors with $\kappa>1 / \sqrt{2}$, the interactions are repulsive due to the negative normal-superconducting surface energy, leading to the formation of the Abrikosov vortex lattice; ${ }^{2}$ (2) in the recently discovered two-band type- 1.5 superconductors, ${ }^{3}$ with $\kappa_{1}>1 / \sqrt{2}$ and $\kappa_{2}<1 / \sqrt{2}$, the interactions are long-range attractive and short-range repulsive. Under the combination of the two interactions, the flux penetration will form vortex clusters and stripes. ${ }^{4}$ (3) In single-band type-I superconductors with $\kappa<1 / \sqrt{2}$, due to the positive interface energy, the vortex-vortex interactions become long-range repulsive and short-range attractive and fluxoids tend to merge with each other, resulting into the intermediate state (IS) ${ }^{5-7}$

In type-II and type-1.5 superconductors each fluxoid contains one single flux quantum $\Phi_{0}$, while in type-I superconductors the normal domains, since flux is quantized, contain multiple numbers of $\Phi_{0}$. The number of flux quanta bounded in a normal domain in the IS can typically be up to $10^{7}$ (Ref. 8 ) and the structures constructed from the normal domains vary from flux tubes ${ }^{9,10}$ to laminar structures ${ }^{11,12}$ or suprafroth, ${ }^{13}$ depending on various parameters like magnetic field, temperature, pinning, and sample geometries. Despite the intense study on the stability, ${ }^{14-16}$ magnetic and topological hysteresis ${ }^{17-19}$ of the IS in type-I superconductors, little is known about the state of flux tubes. Since the flux tubes are believed to be the basic building blocks of the IS, ${ }^{8,9}$ it is of great fundamental interest and importance to answer the following questions: First, how do the flux tubes interact with each other? It is natural to expect that the interaction between two flux tubes must also happen through quantized flux. However, it has never been confirmed experimentally. Theoretical results have revealed that the nucleation and interaction of flux domains under an applied current are both occurring via the nucleation of individual flux quanta. ${ }^{20}$
Therefore, this gives rise to a second question: Is it possible to have stable flux domains with single flux quantum in the IS of a type-I superconductor? In the 1960s, theoretical work had shown that, in thin enough type-I superconductor films with perpendicular magnetic field, the transition from superconducting to normal state can be type-II-like, ${ }^{21,22}$ and triangular vortex lattices may favor a more energetically stable state. $^{23}$ Various experimental results have also confirmed such a prediction. ${ }^{24,25}$ The critical thickness $d_{c}$, below which single vortices can exist, varies with the material ${ }^{26,27}$ (e.g., $d_{c} \sim 200 \mathrm{~nm}$ for $\mathrm{Pb} ; \sim 110 \mathrm{~nm}$ for In). Very recently, the single flux quantum vortices have been suggested to exist in mesoscopic type-I materials with strong confinement effects. ${ }^{28}$ Engbarth et al. reported the observation of $\Phi_{0}$ vortices in a one-dimensional (1D) type-I $\mathrm{Pb}$ nanowire through local magnetization measurements. ${ }^{29}$ However, in macroscopic samples, $\Phi_{0}$ fluxoids have never been observed either experimentally or reported theoretically. Is it possible to stabilize single-quantum vortices in the intermediate state of a macroscopic type-I superconductor? If not, what would be the minimum possible flux for the IS? It is believed that, due to the connection to the sample edges, the expulsion of lamellae in the IS is continuous, while upon zero-field cooling (ZFC) the flux penetration will be broken up by the geometrical energy barrier, which isolates the flux tubes from the sample edges through a diamagnetic band. ${ }^{7}$ The continuous expulsion of flux provides a possible way to control the vorticity of flux tubes.

In order to shed light on the above questions, here we report on the study of the quantization of the flux tubes in a thick type-I superconducting film by using high resolution scanning Hall probe microscopy (SHPM). The flux tubes are observed in both flux penetration and expulsion processes. All the flux tubes can be well fitted using a monopole model which shows that the observed flux tubes carry an integer number of flux quanta within the experimental error. $\Phi_{0}$ vortices are observed only in the flux expulsion process, while upon flux penetration the minimum flux obtained contains four flux quanta at $T=$ $6.9 \mathrm{~K}$ due to the presence of the surface energy barrier. The integration results of SHPM signal are consistent with the fits using the monopole model. 
The type-I lead film with a dimension of $1 \times 1 \mathrm{~cm}^{2}$ was grown using e-beam evaporation with a thickness of $d=5 \mu \mathrm{m}$. A $10-\mathrm{nm}$ Ge layer is evaporated on top to protect the $\mathrm{Pb}$ surface from oxidation. The detailed description of the sample preparation process can be found in Ref. 30 The superconducting critical temperature of the sample is $7.05 \mathrm{~K}$ as determined from the local ac response at zero field. The SHPM images were obtained using a modified Low Temperature Scanning Hall Probe Microscope from Nanomagnetics Instruments with a temperature resolution better than $1 \mathrm{mK}$ and a field resolution better than $0.1 \mathrm{G}$. The scanned area is $16 \times 16 \mu \mathrm{m}^{2}$, close to the center of the sample. During the experiments, the field is applied perpendicular to the sample surface. A well-known type-II superconductor, $\mathrm{NbSe}_{2}$ single crystal with $T_{c} \sim 7 \mathrm{~K}$, was used as a reference.

To fit the fluxoid magnetic field, a monopole model is often used if the constraint $\left(r^{2}+z^{2}\right) \gg \lambda_{a b}$ is satisfied, ${ }^{31-34}$ where $r$ is the distance from the fluxoid center, $z$ is the height of the Hall probe to the sample surface, and $\lambda_{a b}$ is the penetration depth. The magnetic field perpendicular to the sample surface $B_{z}(r, z)$ is expressed as

$$
B_{z}(r, z)=\frac{\Phi}{2 \pi} \frac{z+\lambda_{a b}}{\left[r^{2}+\left(z+\lambda_{a b}\right)^{2}\right]^{3 / 2}},
$$

where $\Phi$ is the total flux bounded in a fluxoid. According to Ref. 35, the accuracy of the model can be enhanced by averaging over an area representative of the Hall probe active area to account for the convolution of the field over the probe. The integration of Eq. (1) over a square active area of sides $s$ and divided by the area $s^{2}$ gives the following result:

$$
\begin{aligned}
B(x, y, z)= & \frac{\Phi}{2 \pi s^{2}}\left[\left[\frac{1}{2} \arctan \left(\frac{2 x y Z \sqrt{x^{2}+y^{2}+Z^{2}}}{Z^{2}\left(x^{2}+y^{2}+Z^{2}\right)-x^{2} y^{2}}\right)\right.\right. \\
& \left.+\operatorname{Fix}(x, y, Z)]_{x-\frac{s}{2}}^{x+\frac{s}{2}}\right]_{y-\frac{s}{2}}^{y+\frac{s}{2}},
\end{aligned}
$$

where $Z=z+\lambda_{a b}, \operatorname{Fix}(x, y, Z)$ is the self-defined function to eliminate the $\pi / 2$ jump and make the final expression continuous. ${ }^{35}$ For our SHPM, a Hall probe with an active area of $s^{2}=0.4 \times 0.4 \mu \mathrm{m}^{2}$ is used.

Figure 1(a) shows a SHPM image of the intermediate state observed after FC at $H=7 \mathrm{Oe}$ and $T=4.2 \mathrm{~K}$. Seven fluxoids with different sizes are observed in the scanned area. Cross sections (solid symbols) of fluxoid I and II as depicted by the white lines in Fig. 1(a) are demonstrated in Fig. 1(b). The best fitting using Eq. (2) gives (I) $Z=1.237 \mu \mathrm{m}, \Phi=$ $5.8 \pm 0.2 h / 2 e$ and (II) $Z=1.266 \mu \mathrm{m}, \Phi=11.9 \pm 0.2 \mathrm{~h} / 2 e$, respectively. For comparison, we also show, in Fig. 1(b), the cross sections of a vortex and an antivortex observed in a reference $\mathrm{NbSe}_{2}$ single crystal at $T=4.2 \mathrm{~K}$. The fitting gives an average $\Phi=0.95 \pm 0.2 h / 2 e$.

We also use integration of the SHPM signal to determine the total flux bounded in each fluxoid. It should be noted that the integrated value is dependent on the effective area chosen for the fluxoid. An integration of a flux quantum, simulated using the monopole model with $Z=1.3 \mu \mathrm{m}, s=0.4 \mu \mathrm{m}$, over an infinite area gives $\Phi_{0}=h / 2 e$. However, for an SHPM image with the typical frame size of $16 \times 16 \mu \mathrm{m}^{2}$, the integration
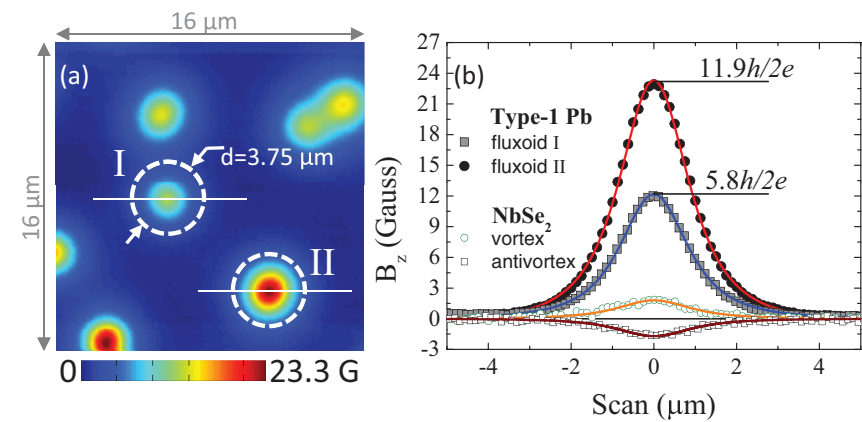

FIG. 1. (Color online) (a) SHPM image obtained after FC at $H=$ $7 \mathrm{Oe}$ and $T=4.2 \mathrm{~K}$ for type-I lead film. The dashed circles show the area chosen for integration. (b) $2 \mathrm{D}$ fit (solid lines) to the data (solid symbols) from cross sections of the flux tubes as shown by the white lines in the SHPM image of Fig. 1(a). The fits give (I) $Z=1.237 \mu \mathrm{m}$ and $\Phi=5.8 \pm 0.2 h / 2 e$ and (II) $Z=1.266 \mu \mathrm{m}$ and $\Phi=11.9 \pm 0.2 h / 2 e$. The open symbols show the cross sections of a vortex and an antivortex observed in a reference $\mathrm{NbSe}_{2}$ single crystal.

of the whole scanned area for the same flux quantum only gives $0.84 h / 2 e$. Normally, in both IS and the mixed state of type-II material, there are more than one fluxoids in each SHPM image. Therefore, to avoid the additional fields from other fluxoids, we can only integrate the signal over a finite area which is very close to the target fluxoid. It can be deduced from Eq. (2) that for fluxoids simulated using the monopole model with the same parameters of $Z$ and $s$, the integrated flux over a constant circular area with radius $r$ from the vortex center is proportional to the total flux $\Phi$, e.g., with $\Phi=$ $L \times h / 2 e, Z=1.25 \mu \mathrm{m}, s=0.4 \mu \mathrm{m}, 2 r=3.75 \mu \mathrm{m}$, the integration always gives $\Phi_{\text {integrate }}=L \times 0.46 h / 2 e$, where $L$ is the vorticity of each fluxoid. Therefore, we can define an effective flux quantum $\phi_{0}=0.46 h / 2 e$. In our case, given a fixed Hall cross area, the parameters that affect the effective flux quantum are the $Z$ value and the area chosen for integration. In the remaining part of this Letter, the integrations are all based on the same area with $2 r=3.75 \mu \mathrm{m}$ as shown by the dashed circle in Fig. 1(a).

For the fluxoid I and II in Fig. 1(a) with the $Z$ values determined from fitting, the effective flux quantum is deduced as $\phi_{0}{ }^{\mathrm{I}}=0.44 h / 2 e$ and $\phi_{0}{ }^{\mathrm{II}}=0.43 h / 2 e$, respectively. Integrating the SHPM signal yields $\Phi_{\mathrm{I}}=5.9 \pm 0.12 \phi_{0}{ }^{\mathrm{I}}$ and $\Phi_{\mathrm{II}}=$ $11.7 \pm 0.12 \phi_{0}{ }^{\mathrm{II}}$, which are consistent with the $2 \mathrm{D}$ monopole model fittings. The quoted errors include conservative estimates of uncertainties from the estimate of the background arising from the noise.

To study the stability and temperature dependence of flux tubes, we recorded the SHPM images by first performing FC to $6 \mathrm{~K}$ at $H=3 \mathrm{Oe}$ [Fig. 2(1)] and then progressively increasing the temperature to very close to $T_{c}$ [Fig. 2(6)]. Six flux tubes, as shown in Fig. 2(1), are observed with red bright colors representing high fields and dark blue colors showing the Meissner state. Compared to Fig. 2(1) it is clear that, with increasing temperature, the intensity of the flux tubes decreases, as reflected by the shading of the color from bright red to yellow, due to the expanding of the flux tube diameter, or in another way, the reduction of the condensate strength. 

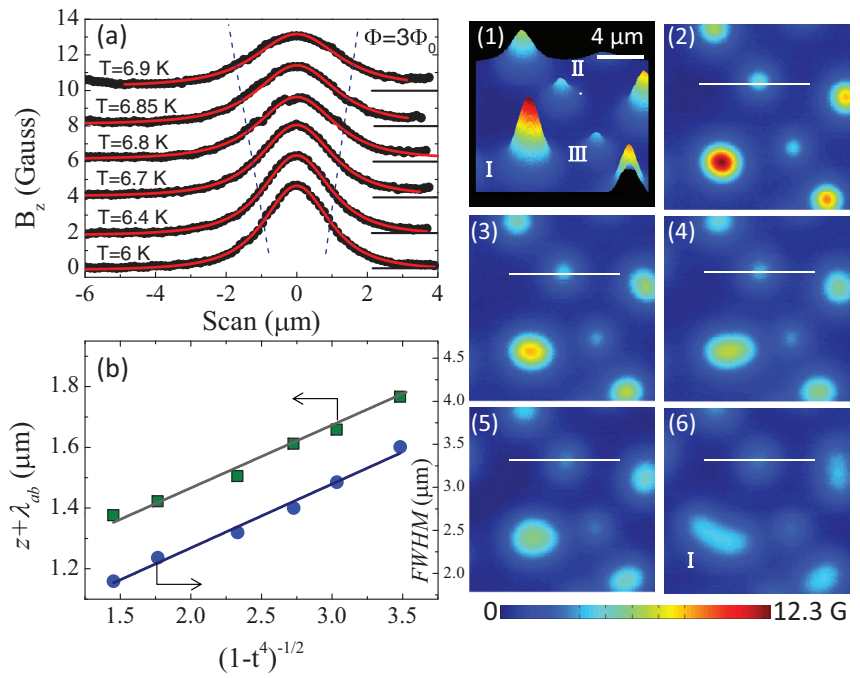

FIG. 2. (Color online) (a) Cross sections of flux tube II, as indicated by the horizontal white lines in the insets. The data are offset by $2 \mathrm{G}$ for clarity. The red lines are $2 \mathrm{D}$ fits to the cross sections with $\Phi$ set at $3 \Phi_{0}$. The distance between the dashed lines shows the expanding full width at half maximum (FWHM) with increasing temperature. (b) $z+\lambda_{a b}$ (squares) and FWHM (circles) as a function of reduced temperature; the solid lines are linear fits to the data. SHPM images obtained by first performing FC at $H=3$ Oe and $T=6 \mathrm{~K}$ (1), and then increasing temperature to (2) $6.4 \mathrm{~K}$, (3) $6.7 \mathrm{~K}$, (4) $6.8 \mathrm{~K}$, (5) $6.85 \mathrm{~K}$, and (6) $6.9 \mathrm{~K}$.

However, the positions of the flux tubes remain unchanged with temperature cycling.

To interpret the spreading of the flux tubes, we show in Fig. 2(a) the cross sections of fluxoid II at various temperatures. The fit and integration for fluxoid II at $T=6 \mathrm{~K}$ yield vorticity corresponding to three flux quanta. Then we fit the cross sections of fluxoid II at various temperatures using Eq. (2) with $\Phi$ fixed at $3 h / 2 e$. Below $T=6.9 \mathrm{~K}$, the cross sections can be well fitted, giving $z+\lambda_{a b}$ from $1.37 \mu \mathrm{m}$ at $6 \mathrm{~K}$ to $1.66 \mu \mathrm{m}$ at $6.85 \mathrm{~K}$. At $T=6.9 \mathrm{~K}$ the fitting curve starts to deviate from the data, probably because of the deformation of the flux tube [also seen for fluxoid I in Fig. 2(6)] or the invalidity of the model very close to $T_{c}$. Figure 2(b) shows the value of $z+\lambda_{a b}$ and the FWHM of the cross sections as a function of reduced temperature. For a superconductor with an isotropic energy gap, the temperature dependence of magnetic penetration depth is proportional to $\left(1-t^{4}\right)^{-1 / 2}$, where $t=T / T_{c}$. The observed linear dependence of $z+\lambda_{a b}$ and FWHM vs $\left(1-t^{4}\right)^{-1 / 2}$ indicates that the spreading of flux tubes mainly arises from the increase of the penetration depth with temperature. Similar behavior is also observed for vortices in the mixed state of high $T_{c}$ materials. ${ }^{33}$

In the IS of type-I superconductors, the energy of singly quantized vortices is larger than the energy of multiply quantized flux tubes, which favors an unstable state if it is composed of $\Phi_{0}$ fluxoids. So it is natural to ask what will be the minimum flux tube of the IS. In Fig. 3 we show SHPM images measured at $6.5 \mathrm{~K}$ after $\mathrm{FC}$ under various magnetic fields. For clarity, the images are presented using a high contrast color map. Above $H=0.2$ Oe, the fluxoid, indicated by the dashed circle, nucleates in the scanned area. The vorticity of

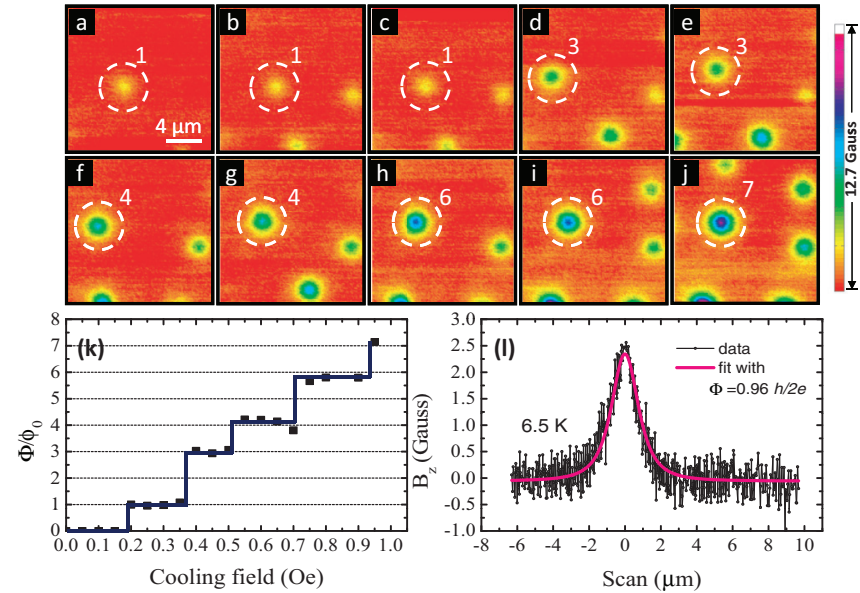

FIG. 3. (Color online) SHPM images observed after FC to $6.5 \mathrm{~K}$ at various magnetic fields: (a) $0.25 \mathrm{Oe}$; (b) $0.3 \mathrm{Oe}$; (c) $0.35 \mathrm{Oe}$; (d) $0.4 \mathrm{Oe}$; (e) $0.5 \mathrm{Oe}$; (f) $0.6 \mathrm{Oe}$; (g) 0.65; (h) $0.75 \mathrm{Oe}$; (i) $0.9 \mathrm{Oe}$; (j) $1.2 \mathrm{Oe}$. The dashed white circles show the positions of the fluxoids with the numbers on top indicating their vorticity. (k) Vorticity as a function of applied field. The solid line is a guide to the eyes. (1) Cross sections of five fluxoids observed below 0.4 Oe. The best fit (solid line) gives $\Phi=0.96 h / 2 e$.

the fluxoid, shown by the number near the circle, is determined from both fitting and integration. Figure $3(\mathrm{k})$ shows the result of integration for the flux tube observed at different cooling fields. Below $H=0.2$ Oe, no fluxoid is formed. Single flux quantum is observed in the field range of $0.2 \leqslant H<0.4$ Oe. With increasing the cooling field, the vorticity increases up to $L=7$ at $H=0.95$ Oe. In Fig. 3(1) we show cross sections of seven singly quantized fluxoids. The fit by the monopole model yields $\Phi=0.96 h / 2 e$ as shown by the solid line.

The observation of single flux quantum in such a macroscopic sample is quite surprising, and to the best of our knowledge, this is the first time that single flux quantum is experimentally observed in the IS of a macroscopic type-I superconductor. It should also be noted that the single flux quantum observed here has to be stabilized by a different mechanism from those considered in Refs. 28 and 29, where the formation of individual vortices is due to the strong geometrical confinement, and individual vortices are observed in both flux penetration and expulsion processes for the type-I nanowires. ${ }^{29}$ In macroscopic type-I superconductors, the IS is a result of the competition between the magnetic energy that favors the formation of small normal domains and the positive surface energy that tends to form large domains. It has been argued that in mesoscopic type-I samples a third interaction, provided by the confinement effect of the sample's boundaries, is responsible for the stabilization of single-quanta fluxoids. ${ }^{28}$ However, in our film (with lateral dimension $1 \times 1 \mathrm{~cm}$ and $d=$ $5 \mu \mathrm{m}$ ), the geometrical confinement effect on the flux tubes has to be very weak. We argue that the stabilization of singly quantized fluxoids in a macroscopic type-I superconductor is possible due to the extra interaction introduced in the system by a weak collective pinning landscape, playing a similar role as the extra interaction introduced in mesoscopic samples due to geometry confinement. ${ }^{36,37}$ 

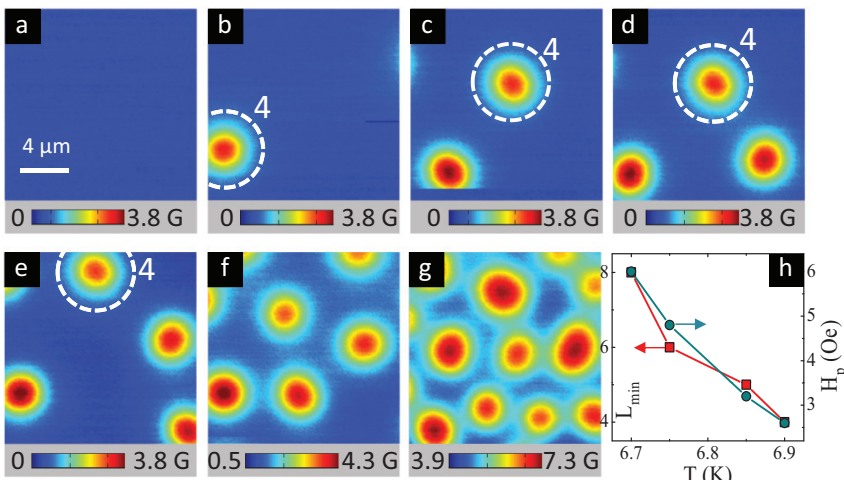

FIG. 4. (Color online) SHPM images observed after first performing ZFC to $6.9 \mathrm{~K}$ and subsequently increasing the magnetic field to (a) $2 \mathrm{Oe}$; (b) $2.6 \mathrm{Oe}$; (c) $2.8 \mathrm{Oe}$; (d) $3 \mathrm{Oe}$; (e) $3.4 \mathrm{Oe}$; (f) $4 \mathrm{Oe}$; (g) 7.6 Oe. The minimum flux tube observed contains four flux quanta as shown by the dashed circles with the vorticity indicated beside it. (h) Penetration field $H_{p}$ (circles) and the vorticity of minimum fluxoid (squares) as a function of temperature.

In Fig. 4 we show the observed flux tubes induced through the flux penetration process after $\mathrm{ZFC}$ to $6.9 \mathrm{~K}$. Below $H=$ $2 \mathrm{Oe}$, the sample remains in the Meissner state [Fig. 4(a)]. At $H=2.6 \mathrm{Oe}$, the first flux tube is observed in the scanned area [Fig. 4(b)]. With increasing field, more and more flux tubes penetrate the scanned area [Figs. 4(c)-4(g), and eventually they will merge and start forming stripes. ${ }^{38}$ The minimum flux tube observed at $T=6.9 \mathrm{~K}$, as shown by the dashed circles, contains four flux quanta. Looking at the temperature dependence of $L_{\text {min }}$, we have observed that $L_{\min }$ increases with decreasing temperature as shown in Fig. 4(h). These observations are in agreement with the impeded flux penetration scenario. According to Refs. 7 and 20, the penetration of magnetic flux in the intermediate state of a type-I superconductor is impeded. That is, the vortices will first accumulate at the sample border to form flux tubes; once the flux tubes contain a large enough amount of magnetic flux as to overcome the surface barrier, they will burst into the sample interior pushed by the Meissner currents. Therefore, our data give an indirect evidence for the impeded flux penetration. As observed, at lower temperatures, due to the increase of the surface energy barrier, the minimum flux tube is expected to contain multiple flux quanta to overcome the energy barrier. Figure 4(h) shows the penetration field $H_{p}$ as a function of temperature. It is clear that with decreasing temperature, the penetration field increases due to the enhanced surface energy barrier. Therefore, the minimum fluxoid also increases. We notice that the flux tubes may contain a slightly different number of flux quanta; this is probably due to the presence of small edge imperfections which yield slightly different energy barriers.

In our experiments, we have not observed any singly quantized flux tube by flux penetration, but it cannot be ruled out at high temperatures, especially close to the normalintermediate transition. This is a process that still needs further investigation. In addition, our data give further evidence that the origin of the nucleation process of single flux quanta in a large film is different from that in the mesoscopic samples. ${ }^{29}$
The diameter of the flux tubes as a function of external field is a subject of intense debates for many years. While a currentloop model predicts a monotonous increase with external field, ${ }^{9,39}$ experiments have shown contradictory results. ${ }^{7,8}$ By quantifying the flux tubes, we have found that the flux tube diameter depends on two parameters: vorticity and the penetration depth $\lambda(T)$. At fixed temperature, the vorticity of flux tubes is mainly determined by the surface energy barrier, and all the flux tubes contain a slightly different number of flux quanta and the average diameter of the flux tubes through flux penetration remains constant with increasing external field in the field range $0.1 \sim 0.3 f$, where $f=H / H_{c}$ and $H_{c}=23$ Oe at $6.9 \mathrm{~K}$. Only at sufficiently high fields, the density of flux tubes increases so much that some of them merge to form bigger ones $[H=7.6$ Oe for Fig. 4(g)] or even large normal domains.

As discussed above, the flux expulsion yields flux tubes with various vorticity while flux penetration results in flux tubes with similar vorticity. It is of interest to know that, in the critical state with positive flux tubes nucleated from flux expulsion and negative flux tubes by flux penetration, i.e., the giant vortex-antivortex state (gv-av), whether the annihilation process is different from that in type-II superconductors.

The critical state of the IS is achieved by first performing FC with an external magnetic field $H$ to a temperature just below $T_{c}$ and then decreasing $H$ progressively. With decreasing field, flux tubes with positive magnetic field (giant vortices) will nucleate as the case in Fig. 1(a). Close to $-H_{p}=$ $-(1-N) H_{c}(T)$, where $N$ is the demagnetization factor due to the sample geometry and $H_{c}(T)$ is the thermodynamic critical field, flux tubes with opposite directions (giant antivortices) begin to penetrate into the sample, leading to the coexistence of the giant vortex-antivortex state. Figure 5(a) shows the gv-av state observed at $T=6.8 \mathrm{~K}$ and $H=-4.47 \mathrm{Oe}$. Three flux tubes with different vorticities are observed in the scanned area. To study the stability of the gv-av state, a small perturbation is applied to the vortex state in Fig. 5(a) by further decreasing the external field to -4.52 Oe [Fig. 5(b)]. The negative flux tubes are pushed into the sample center due to the Lorentz force exerted by the Meissner current on them. Since the SHPM images were taken by scanning line by line from bottom to top, we obtain the process of annihilation between one giant antivortex and one giant vortex as shown in Fig. 2(b). In the bottom few lines we can still see the giant vortex seated on the pinning center, while after a few seconds a giant antivortex has moved in and overlapped with it, resulting in a new vortex pattern as shown in Fig. 5(c). It has to be mentioned that due the positive N-S surface energy in type-I superconductors, the vortex interaction between vortex and antivortex is repulsive. ${ }^{40}$ However, the repulsive force is quite weak compared with the Lorentz force, which is also supported by the observation of the annihilation of them as indicated in Fig. 5(b).

Note that the giant antivortex I and III contain significant different intensities. We have used the monopole model to quantify them. As shown, in Fig. 5(d) by the solid lines, the fitting of the cross sections yield $4 \Phi_{0}, 2.2 \Phi_{0}$, and $\Phi_{0}$ for flux tube a-I, c-I, and c-III, respectively. This indicates that the original giant antivortex, before annihilating with a-I, contains $6 \Phi_{0}$, consistent with the minimum fluxoid vorticity as a function of temperature in Fig. 4(h). The fact that fluxoid c-III only contains one flux quantum is quite surprising since 

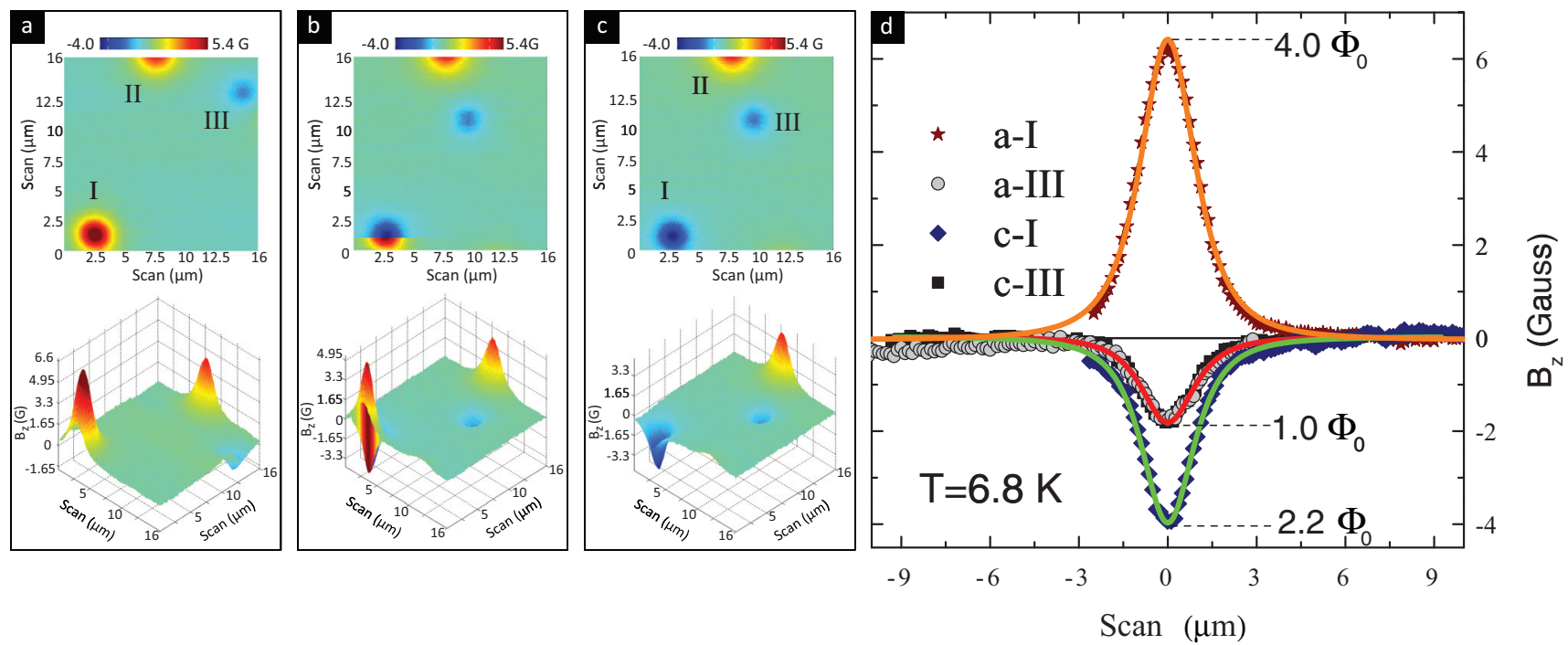

FIG. 5. (Color online) 2D (upper panel) and 3D (lower panel) SHPM images measured after FC with $H=7$ Oe to $6.8 \mathrm{~K}$, then decreasing field to (a) -4.47 Oe and (b) -4.52 , respectively. (c) Image taken five minutes later than (b) with the same parameters. (d) Cross sections (symbols) of the flux tubes in (a) and (c). The solid lines are the fitting curves using the monopole model.

$\Phi_{0}$ flux tube has never been observed through flux penetration process even at temperatures very close to $T_{c}$. Here, it is very unlikely that c-III penetrated directly from the border into the sample interior. The most reasonable case is that, on the way from the sample border to the interior, one giant antivortex annihilates with one or more pinned giant vortex, resulting in the formation of the single quantum fluxoid. The annihilation process provides another way to form $\Phi_{0}$ vortices in the IS of type-I superconductors. There is a significant difference between c-III and the $\Phi_{0}$ vortices observed through flux expulsion process: The nucleation process is totally different where c-III originates from the penetrated flux tube through the sample border instead of flux expulsion. Moreover, since c-III is pushed into the scanned area (sample center) from the sample border, it gives a strong evidence that single quantum vortices are thermodynamically stable in type-I superconductors.

In summary, we have provided direct experimental evidence for the presence of stable single quantized vortices in the intermediate state of a macroscopic type-I superconductor. Contrary to the mesoscopic case, where the stabilization of the single quantum vortex was predicted due to the strong geometrical confinements, our findings in a macroscopic sample suggests that the presence of very weak collective pinning may also stabilize single flux quanta in type-I superconductors. Therefore, we believe that our results will certainly stimulate the development of adequate theoretical descriptions. We also have shown that the average size of flux tubes is found to be determined by the vorticity and the temperature dependence of penetration depth, and only multiquanta flux tubes are observed through the flux penetration process due to the existence of the surface energy barrier, which gives an evidence for the impeded flux penetration process as predicted theoretically. Moreover, by exploiting the analogies of the intermediate state of a type-I superconductor with other physical systems, the present findings can be important for the relevant research on ferrofluids, amphiphilic monolayer, and chemical reaction-diffusion systems, ${ }^{41}$ or even neutron stars. ${ }^{42}$

This work is supported by the FWO projects, the Methusalem Funding by the Flemish Government and the MP1201 COST Action.
*Victor.Moshchalkov@ fys.kuleuven.be

${ }^{1}$ B. S. Deaver, Jr. and W. M. Fairbank, Phys. Rev. Lett. 7, 43 (1961).

${ }^{2}$ H. F. Hess, R. B. Robinson, R. C. Dynes, J. M. Valles, and J. V. Waszczak, Phys. Rev. Lett. 62, 214 (1989).

${ }^{3}$ V. Moshchalkov, M. Menghini, T. Nishio, Q. H. Chen, A. V. Silhanek, V. H. Dao, L. F. Chibotaru, N. D. Zhigadlo, and J. Karpinski, Phys. Rev. Lett. 102, 117001 (2009).

${ }^{4}$ J. Gutierrez, B. Raes, A. V. Silhanek, L. J. Li, N. D. Zhigadlo, J. Karpinski, J. Tempere, and V. V. Moshchalkov, Phys. Rev. B 85, 094511 (2012).

${ }^{5}$ A. G. Meshkovskii and A. I. Shal'nikov, Zh. Eksp. Teor. Fiz. 17, 851 (1947).
${ }^{6}$ B. V. Makei, JETP 7, 217 (1958).

${ }^{7}$ V. Jeudy, C. Gourdon, and T. Okada, Phys. Rev. Lett. 92, 147001 (2004).

${ }^{8}$ J. R. Hoberg and R. Prozorov, Phys. Rev. B 78, 104511 (2008).

${ }^{9}$ R. N. Goren and M. Tinkham, J. Low Temp. Phys. 5, 465 (1971).

${ }^{10}$ A. Cebers, C. Gourdon, V. Jeudy, and T. Okada, Phys. Rev. B 72, 014513 (2005).

${ }^{11}$ L. D. Landau, Zh. Eksp. Teor. Fiz. 7, 371 (1937).

${ }^{12}$ R. P. Huebener, R. T. Kampwirth, and V. A. Rowe, Cryogenics 12, 100 (1972). 
${ }^{13}$ R. Prozorov, A. F. Fidler, J. R. Hoberg, and P. C. Canfield, Nature Phys. 4, 327 (2008).

${ }^{14}$ R. Prozorov, Phys. Rev. Lett. 98, 257001 (2007).

${ }^{15}$ M. Menghini and R. J. Wijngaarden, Phys. Rev. B 75, 014529 (2007).

${ }^{16}$ M. Seul, Europhys. Lett. 28, 557 (1994).

${ }^{17}$ R. Prozorov, R. W. Giannetta, A. A. Polyanskii, and G. K. Perkins, Phys. Rev. B 72, 212508 (2005).

${ }^{18}$ S. Velez, C. Panades-Guinart, G. Abril, A. Garcia-Santiago, J. M. Hernandez, and J. Tejada, Phys. Rev. B 78, 134501 (2008).

${ }^{19}$ C. Gourdon, V. Jeudy, and A. Cebers, Phys. Rev. Lett. 96, 087002 (2006).

${ }^{20}$ G. R. Berdiyorov, A. D. Hernandez-Nieves, M. V. Milosevic, F. M. Peeters, and D. Dominguez, Phys. Rev. B 85, 092502 (2012).

${ }^{21}$ M. Tinkham, Phys. Rev. 129, 2413 (1963).

${ }^{22}$ K. Maki, Ann. Phys. (NY) 34, 363 (1965).

${ }^{23}$ G. Lasher, Phys. Rev. 154, 345 (1967).

${ }^{24}$ H. Boersch, U. Kunze, B. Lischke, and W. Rodewald, Phys. Lett. A 44, 273 (1973).

${ }^{25}$ G. J. Dolan and J. Silcox, Phys. Rev. Lett. 30, 603 (1973).

${ }^{26}$ S. Onori and A. Rogani, Physica B 132, 217 (1985).

${ }^{27}$ O. Singh and A. E. Curzon, Cryogenics 15, 665 (1975).

${ }^{28}$ G. R. Berdiyorov, A. D. Hernandez, and F. M. Peeters, Phys. Rev. Lett. 103, 267002 (2009).
${ }^{29}$ M. A. Engbarth, S. J. Bending, and M. V. Milosevic, Phys. Rev. B 83, 224504 (2011).

${ }^{30}$ Y. Bruynseraede, K. Temst, E. Osquiguil, C. Van Haesendonck, A. Gilabert, and I. K. Schuller, Physica Scripta 42, 37 (1992).

${ }^{31}$ J. Pearl, J. Appl. Phys. 37, 4139 (1966).

${ }^{32}$ A. M. Chang et al., Appl. Phys. Lett. 61, 1974 (1992).

${ }^{33}$ J. C. Wynn, D. A. Bonn, B. W. Gardner, Y. J. Lin, R. Liang, W. N. Hardy, J. R. Kirtley, and K. A. Moler, Phys. Rev. Lett. 87, 197002 (2001).

${ }^{34}$ T. Nishio, Q. Chen, W. Gillijns, K. D. DeKeyser, K. Vervaeke, and V. V. Moshchalkov, Phys. Rev. B 77, 012502 (2008).

${ }^{35}$ J. W. Guikema, Ph.D. thesis, Stanford University, 2004.

${ }^{36}$ M. Baert, V. V. Metlushko, R. Jonckheere, V. V. Moshchalkov, and Y. Bruynseraede, Phys. Rev. Lett. 74, 3269 (1995).

${ }^{37}$ V. V. Moshchalkov, M. Baert, V. V. Metlushko, E. Rosseel, M. J. Van Bael, K. Temst, Y. Bruynseraede, and R. Jonckheere, Phys. Rev. B 57, 3615 (1998).

${ }^{38}$ J. Ge, J. Gutierrez, B. Raes, J. Cuppens, and V. V. Moshchalkov, New J. Phys. 15, 033013 (2013).

${ }^{39}$ A. T. Dorsey and R. E. Goldstein, Phys. Rev. B 57, 3058 (1998).

${ }^{40}$ V. R. Misko, V. M. Fomin, J. T. Devreese, and V. V. Moshchalkov, Phys. Rev. Lett. 90, 147003 (2003).

${ }^{41}$ M. Seul and D. Andelman, Science 267, 476 (1995).

${ }^{42}$ K. B. W. Buckley, M. A. Metlitski, and A. R. Zhitnitsky, Phys. Rev. Lett. 92, 151102 (2004). 